Check for updates

1 Chatfield Health Care, London, UK

2 Department of Respiratory Medicine, UCLH NHS Foundation Trust, London, UK

3 Warwick Medical School, University of Warwick, Coventry, UK

Correspondence to W Shah waqaar.shah@nhs.net Cite this as: BMJ 2021;372:n136 http://dx.doi.org/10.1136/bmj.n136 Published: 22 January 2021

\title{
GUIDELINES
}

\section{Managing the long term effects of covid-19: summary of NICE, SIGN, and RCGP rapid guideline}

\author{
Waqaar Shah, ${ }^{1}$ Toby Hillman, ${ }^{2}$ E Diane Playford, ${ }^{3}$ Lyth Hishmeh
}

\section{What you need to know}

- The likelihood of developing long term effects of covid-19 is not thought to be related to the severity of the acute infection

- The most common symptoms of long term covid-19 are fatigue and breathlessness. Symptoms may be singular, multiple, constant, transient, or fluctuating, and can change in nature over time

- Offer a chest radiograph by 12 weeks after acute covid-19 if the person has not had one already and has continuing respiratory symptoms

For a proportion of people covid-19 leads to long term effects that can have a significant impact on quality of life. According to the Office for National Statistics, around one in five people testing positive for covid-19 exhibit symptoms for a period of five weeks or more. ${ }^{1}$ This presents challenges for determining best-practice standards of care. As yet, no commonly agreed clinical definition of long term covid-19 exists, nor a clear definition of treatment pathway. To assist clinicians, the National Institute for Health and Care Excellence (NICE), the Scottish Intercollegiate Guidelines Network (SIGN), and the Royal College of General Practitioners (RCGP) have developed the "COVID-19 rapid guideline: managing the long term effects of COVID-19." It covers care for people with signs and symptoms that continue for more than four weeks, and which developed during or after an infection consistent with covid-19, and which are not explained by alternative diagnoses.

The guideline provides clinical definitions of the effects of covid-19 at different times and provides advice on diagnosis and management based on the best available evidence and the knowledge and experience of the expert panel. It will be subject to a "living" approach, which means that targeted areas of the guideline will be reviewed weekly and updated in response to emerging evidence and evolving expert experience. This article summarises the guideline recommendations as published on 18 December 2020, with particular emphasis on primary care. Updates are available on the NICE website. ${ }^{2}$

\section{Recommendations}

The guideline is part of a series of rapid guidelines on covid-19. ${ }^{3}$ Available evidence was of poor quality, unsurprisingly given the novel nature of this condition, and recommendations were made mainly based on expert opinion.

\section{Definitions}

The guideline defines acute covid-19, ongoing symptomatic covid-19, and post-covid-19 syndrome, according to duration of symptoms. The guideline acknowledges common usage of "long covid," but the panel felt discrete, time-bound terms would better facilitate access to support, provide the basis for service planning, and enable clinical datasets to be established for monitoring and research. Box 1 gives definitions.

\section{Box 1: Covid-19 definitions}

- Acute covid-19 infection-Signs and symptoms of covid-19 for up to four weeks

- Ongoing symptomatic covid-19-Signs and symptoms of covid-19 present from four weeks and up to 12 weeks

- Post-covid-19 syndrome-Signs and symptoms that develop during or after an infection consistent with covid-19, present for more than 12 weeks and are not attributable to alternative diagnoses

Identifying people with ongoing symptomatic covid-19 or post-covid-19 syndrome

The guideline makes recommendations for healthcare professionals caring for people who have had suspected or confirmed acute covid-19 and present to any healthcare setting, irrespective of whether they were hospitalised or had a positive or negative SARS-CoV-2 test (polymerase chain reaction, antigen, or antibody). The guideline emphasises providing information to empower people to understand their symptoms, and to recognise when to seek help.

- Give people who have had suspected or confirmed acute covid-19 (and their families or carers, as appropriate) advice and written information on

- the most common new or ongoing symptoms after acute covid-19

- what they might expect during their recovery, including that:

recovery time is different for everyone, but for many people symptoms will resolve by 12 weeks

the likelihood of developing ongoing symptomatic covid-19 or post-covid-19 syndrome is not thought to be linked to the severity of their acute covid-19 (including whether they were in hospital) 
if new or ongoing symptoms occur they can change unpredictably, affecting people in different ways at different times

- how to self-manage ongoing symptomatic covid-19 or post-covid-19 syndrome

- symptoms to look out for that mean they should contact their healthcare professional

- who to contact if they are worried about new, ongoing, or worsening symptoms, especially if they have them more than four weeks after the start of acute covid-19.

- For people who are concerned about new or ongoing symptoms four weeks or more after acute covid-19, offer an initial consultation and use shared decision making to discuss and agree with the person whether the meeting should be by video, phone, or in person.

Support people in underserved or vulnerable groups to access assessment and care by (for example) raising awareness about the long term effects of covid-19, and providing extra time or additional support (such as an interpreter or advocate) during consultations. Consider follow-up for people in vulnerable groups who have self-managed in the community after suspected or confirmed acute covid-19.

\section{Assessing people with new or ongoing symptoms after acute covid-19}

The most common symptoms are fatigue and breathlessness. Other possible symptoms are listed in box 2. Symptoms may be singular, multiple, constant, transient, or fluctuating, and can change in nature over time. Assessment should include physical, cognitive, psychological, and psychiatric symptoms, as well as functional abilities.

\section{Box 2: Possible symptoms after acute covid-19}

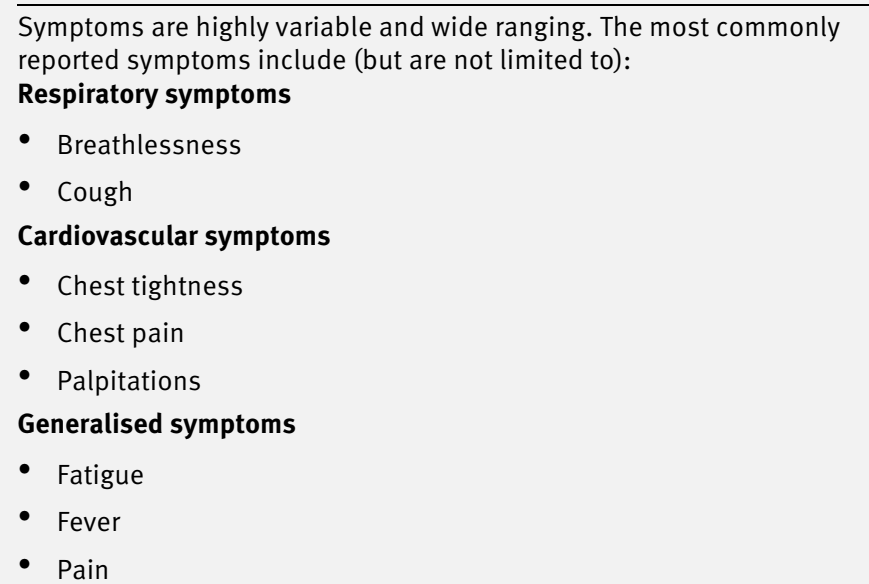

\section{Neurological symptoms}

- Cognitive impairment ("brain fog," loss of concentration or memory issues)

- Headache

- Sleep disturbance

- Peripheral neuropathy symptoms (pins and needles and numbness)

- Dizziness

- Delirium (in older populations)

\section{Gastrointestinal symptoms}

- Abdominal pain
- Nausea

- Diarrhoea

- Anorexia and reduced appetite (in older populations)

Musculoskeletal symptoms

- Joint pain

- Muscle pain

Psychological/psychiatric symptoms

- Symptoms of depression

- Symptoms of anxiety

Ear, nose, and throat symptoms

- Tinnitus

- Earache

- Sore throat

- Loss of taste and/or smell

Dermatological

- Skin rashes

- Include in the comprehensive clinical history:

- history of suspected or confirmed acute covid-19

- the nature and severity of previous and current symptoms

- timing and duration of symptoms since the start of acute covid-19

- history of other health conditions.

- Discuss how the person's life and activities, for example their work or education, mobility, and independence, have been affected by ongoing symptomatic covid-19 or suspected post-covid-19 syndrome.

- Discuss the person's experience of their symptoms and ask about any feelings of worry or distress. Listen to their concerns with empathy and acknowledge the impact of the illness on their day-to-day life, for example, activities of daily living, feelings of social isolation, work and education, and wellbeing.

- Do not predict whether a person is likely to develop post-covid-19 syndrome based on whether they had certain symptoms (or clusters of symptoms) or were in hospital during acute covid-19.

- When investigating possible causes of a gradual decline, deconditioning, worsening frailty or dementia, or loss of interest in eating and drinking in older people, bear in mind that these can be signs of ongoing symptomatic covid-19 or suspected post-covid-19 syndrome.

\section{Investigation and referral}

Covid-19 may cause complications such as myocarditis and postural hypotension. However, not all symptoms will be related to covid-19. Investigations serve to rule out serious or urgent complications, evaluate symptoms secondary to ongoing symptomatic covid-19 or post-covid-19 syndrome, or to look for new, unrelated diagnoses. No one set of investigations and tests would be suitable for everyone because of the wide range of symptoms and severity.

- Offer blood tests, which may include a full blood count, kidney and liver function, $C$ reactive protein, ferritin, B-type natriuretic peptide, and thyroid function. 
- Offer a chest radiograph by 12 weeks after acute covid-19 if the person has not had one already and has continuing respiratory symptoms. Chest radiography appearances alone should not determine the need for referral for further care. Be aware that a plain chest radiograph may not be sufficient to rule out lung disease.

- If appropriate, offer an exercise tolerance test suited to the person's ability (for example the 1 minute sit-to-stand test). During the exercise test, record level of breathlessness, heart rate, and oxygen saturation. Follow an appropriate protocol to carry out the test safely.

- For people with postural symptoms, for example palpitations or dizziness on standing, carry out lying and standing blood pressure and heart rate recordings ( 3 minute active stand test, or 10 minutes if you suspect postural tachycardia syndrome, or other forms of autonomic dysfunction).

- Refer people with ongoing symptomatic covid-19 or suspected post-covid-19 syndrome urgently to the relevant acute services if they have signs or symptoms that could be caused by an acute or life threatening complication, including (but not limited to):

- severe hypoxaemia or oxygen desaturation on exercise

- signs of severe lung disease

- cardiac chest pain

- multisystem inflammatory syndrome (in children).

- After ruling out acute or life threatening complications and alternative diagnoses, consider referring people to an integrated multidisciplinary assessment service (if available) any time from four weeks after the start of acute covid-19.

\section{Planning care}

- After the holistic assessment, use shared decision making to discuss and agree with the person (and their family or carers, if appropriate) what support and rehabilitation they need and how this will be provided. This should include:

- advice on self-management, with the option of supported self-management, and one of the following, depending on clinical need and local pathways:

support from integrated and coordinated primary care, community, rehabilitation, and mental health services

$\checkmark$ referral to an integrated multidisciplinary assessment service

$\square$ referral to specialist care for specific complications.

- When discussing with the person the appropriate level of support and management:

- think about the overall impact their symptoms are having on their life, even if each individual symptom alone may not warrant referral

- look at the overall trajectory of their symptoms, taking into account that symptoms often fluctuate and recur, so they might need different levels of support at different times.

\section{Management}

The guideline offers an overview of self-management and supported self-management. Patient organisations and online support groups can help support self-management.

- Give advice and information on self-management to people with ongoing symptomatic covid-19 or post-covid-19 syndrome, starting from their initial assessment. This should include:

- ways to self-manage their symptoms, such as setting realistic goals

- who to contact if they are worried about their symptoms or they need support with self-management

- sources of advice and support, including support groups, social prescribing, online forums, and apps

- how to get support from other services, including social care, housing, and employment, and advice about financial support

- information about new or continuing symptoms of covid-19 that the person can share with their family, carers, and friends.

- Support people in discussions with their employer, school, or college about returning to work or education, for example by having a phased return.

Based on their experience, the guideline panel agreed that symptom diaries and symptom tracking apps can be helpful for self-monitoring. The evidence for different symptom tracking apps was not reviewed, so the panel could not recommend a specific product. The NHS website "Your COVID Recovery"4 was highlighted as a potential source of reliable, up-to-date information and support.

The panel agreed that multidisciplinary rehabilitation teams should work with people to make a plan for their rehabilitation once any symptoms that could affect the safety of rehabilitation have been investigated. Physical, psychological, and psychiatric aspects of rehabilitation should be addressed, and management of fatigue should be a key component of this. The evidence showed that breathlessness, fatigue, and "brain fog" are among the most commonly reported long term symptoms, so support for these should be part of the person's rehabilitation plan.

\section{Follow-up and monitoring}

- Agree with the person how often follow-up and monitoring are needed and which healthcare professionals should be involved.

- Using shared decision making, offer people the option of monitoring in person or remotely depending on availability, the person's preference, and whether it is clinically suitable for them.

- Tailor monitoring to the person's symptoms and discuss any changes, including new or worsening symptoms and the effects of these on the person's life and wellbeing.

\section{Sharing information and continuity of care}

The panel found evidence that some people have struggled to access appropriate care, and some had experienced fragmented care.

- Ensure effective information sharing and integrated working by sharing clinical records and plans for care and rehabilitation promptly between services and through multidisciplinary meetings, either virtually or in person. 
- Give the person a copy of their care plans or records to keep, including their discharge letters, clinical records, and rehabilitation plans and prescriptions.

- Include baseline measures as well as ongoing assessments in information shared between services, including when the person is discharged from hospital. For example, resting oxygen saturation and heart rate, and the results of functional assessment.

- Provide continuity of care with the same healthcare professional or team as much as possible, for example, by providing a care co-ordinator or a single point of contact.

\section{Service organisation}

The guideline makes recommendations about the provision of, and access to multidisciplinary services. The panel agreed that some of the common elements, such as integration and multidisciplinary team working, would help provide effective, well organised care for people with ongoing symptomatic covid-19 and post-covid-19 syndrome. As well as ensuring the right breadth of expertise, having a multidisciplinary team with input from other services and a clear referral pathways can prevent disjointed care and people waiting a long time for appointments with multiple specialists.

Differential access may be affected, for example, by age, race, disability, homelessness, language, digital accessibility, socioeconomic factors, and mental health conditions.

- Deploy multidisciplinary clinics (possibly using the "one-stop" clinic model) to assess physical and mental health symptoms and to conduct appropriate investigations.

- For rehabilitation, supply an integrated, multidisciplinary service, based on local need and resources. A range of specialists should be available, especially for expertise in treating fatigue and respiratory symptoms (including breathlessness); additional expertise may be sourced depending on the age and symptoms of the person.

- To ensure seamless care, referral pathways need to be integrated between primary and community care, multidisciplinary rehabilitation services, and specialist services, multidisciplinary assessment clinics, and specialist mental health services.

\section{Guidelines into practice}

- Patients with long term effects of covid-19 have symptoms that resemble a wide range of other conditions. Do we always have this in mind when managing the patient?

- People living with long term effects of covid-19 often experience misunderstanding from relatives, friends, and colleagues. How can we best help them manage the uncertainty associated with this condition, and avoid feeling dismissed or ignored?

\section{How patients were involved in the creation of this article}

Six members of the expert advisory panel involved in creating this guideline had experienced long term effects of covid-19. A lay member of the expert advisory panel is co-author of this paper.

These contributors reported that they sometimes felt dismissed and misunderstood by healthcare professionals when they reported long term symptoms. We considered this insight when preparing the sections of this article on referral and management.
Funding statement: The authors received no funding from any sources.

Contributors and guarantor: The guideline referred in this article was produced by NICE, SIGN and the RCGP. The views expressed in this article are those of the authors and not necessarily those of NICE, SIGN, or the RCGP.

Competing interests: Declaration of interests based on NICE's policy on conflicts of interests (available at https:/www.nice.org.uk/Media/Default/About/Who-we-are/Policies-and-procedures/code-of-practicefor-declaring-and-managing-conflicts-of-interest.pdf). Expert advisory panel members' full statements can be viewed at: https://www.nice.org.uk/guidance/ng188/history

Provenance and peer review: commissioned; not externally peer reviewed.

Office for National Statistics. The prevalence of long COVID symptoms and COVID-19 complications. 2020. www.ons.gov.uk/news/statementsandletters/theprevalenceoflongcovidsymptomsandcovid19complications

2 National Institute for Health and Care Excellence, Royal College of General Practitioners, Healthcare Improvement Scotland SIGN. COVID-19 rapid guideline: managing the long term effects of COVID-19. 2020. www.nice.org.uk/guidance/ng188.

3 National Institute for Health and Care Excellence. Developing NICE guidelines: the manual. Process and methods PMG20. 2014. https://www.nice.org.uk/process/pmg20/resources/appendix-l-interim-process-and-methods-for-guidelines-developed-in-response-to-health-and-social-careemergencies-8779776589/chapter/introduction-and-overview

4 NHS UK. Supporting your recovery after COVID-19. 2020. https://www.yourcovidrecovery.nhs.uk/ 